\title{
Comparison of cilostazol versus ticlopidine following coronary stenting in patients with coronary heart disease: A meta-analysis of randomized controlled trials
}

\author{
FENG-HUAN HU ${ }^{1}, \mathrm{XIN} \mathrm{YI}^{2}$, YUE-JING YANG ${ }^{1}$, SHU-BIN QIAO $^{1}$, \\ YONG-JIAN WU ${ }^{1}$ and JIAN-SONG YUAN ${ }^{1}$
}

\begin{abstract}
${ }^{1}$ State Key Laboratory of Cardiovascular Disease, Coronary Artery Diseases Center, Fuwai Hospital, National Center for Cardiovascular Diseases, Chinese Academy of Medical Sciences and Peking Union Medical College, Beijing 100037; ${ }^{2}$ Department of Cardiovascular Medicine, Beijing Hui People Hospital, Beijing 100054, P.R. China
\end{abstract}

Received April 3, 2013; Accepted June 24, 2013

DOI: 10.3892/etm.2013.1190

\begin{abstract}
Previous studies have shown that the combination of cilostazol and aspirin may be a more effective regimen than ticlopidine plus aspirin in the prevention of late restenosis and acute or subacute stent thrombosis following coronary stenting; however, individually published results are inconclusive. The aim of this meta-analysis was to compare the differences in late restenosis and stent thrombosis between cilostazol plus aspirin and ticlopidine plus aspirin for patients with coronary heart disease (CHD) following coronary stenting. A literature search of Pubmed, Embase, Web of Science and Chinese BioMedicine (CBM) databases was conducted from 1998 to March 1, 2013 and statistical analysis was performed using Stata statistical software, version 12.0. Twelve randomized controlled trials were included in the study, with a total of 2,708 patients with CHD following coronary stenting. The patient population comprised 1,371 patients treated with cilostazol plus aspirin and 1,337 patients treated with ticlopidine plus aspirin. The meta-analysis showed that cilostazol plus aspirin demonstrated a lower rate of restenosis than ticlopidine plus aspirin [odds ratio $(\mathrm{OR})=0.83,95 \%$ confidence interval $(\mathrm{CI})=0.69-0.99, \mathrm{P}=0.047]$. A significant difference was also observed in the average percent diameter stenosis between cilostazol plus aspirin and ticlopidine plus aspirin [standardized weight difference (SMD) $=-0.57,95 \% \mathrm{CI}=-0.92,-0.23$, $\mathrm{P}=0.001)$. However, there were no significant differences in the rates of acute or subacute stent thrombosis between cilo-
\end{abstract}

Correspondence to: Dr Feng-Huan $\mathrm{Hu}$, Department of Cardiovascular Medicine, Cardiovascular Institute and Fuwai Hospital, Chinese Academy of Medical Sciences and Beijing Union Medical College, 167 Beilishi Road, Xicheng, Beijing 100054, P.R. China

E-mail: fwyy_hfh@126.com; hufenghuan@medmail.com.cn

Key words: coronary heart disease, coronary stenting, cilostazol, ticlopidine, meta-analysis stazol plus aspirin and ticlopidine plus aspirin. The present meta-analysis suggests that cilostazol plus aspirin may result in a lower restenosis rate and percent diameter stenosis than ticlopidine plus aspirin for patients with CHD following coronary stenting.

\section{Introduction}

Coronary heart disease (CHD), the most common global cause of morbidity and mortality, is known to consume vast medical resources $(1,2)$. Although coronary stenting is widely used in the treatment of patients with CHD, the high rates of late restenosis and stent thrombosis remain the primary limitations (3). At present, adjunctive antiplatelet therapy has been suggested to reduce the incidence rate of restenosis and stent thrombosis $(4,5)$. In addition, the utilization of antiplatelet agents has been demonstrated to be effective in improving final outcomes (6).

Aspirin, a traditional antiplatelet agent, has been most commonly used for the prevention of ischemic arterial events, including coronary thrombosis; however, it has no impact on restenosis (7). Therefore, the introduction of an effective antiplatelet therapy to be used in combination with aspirin and alternative antiplatelet agents following coronary stenting is urgently required. The importance of antiplatelet therapy with ticlopidine plus aspirin in the prevention of subacute thrombosis following coronary artery stenting has been demonstrated (8). However, the use of ticlopidine presents the risk of serious side-effects, such as neutropenia or thrombocytopenia. Cilostazol is a selective cyclic adenosine monophosphate phosphodiesterase inhibitor that is known to inhibit platelet aggregation and intimal hyperplasia $(9,10)$. In view of the fact that cilostazol use presents the risk of mild adverse side effects, cilostazol may theoretically be a desirable substitute for ticlopidine (11). Thus, the adjunctive use of cilostazol plus aspirin following coronary stenting is becoming a more respected option. Recently, it has been demonstrated that cilostazol is able to prevent thrombosis following coronary stenting, reduce restenosis and improve clinical outcomes (12). Moreover, antiplatelet therapy with cilostazol plus aspirin 
has been shown to be effective in preventing late restenosis and stent thrombosis, with less serious complications (13). However, it has also demonstrated that cilostazol plus aspirin is not able to be statistically distinguished from ticlopidine plus aspirin for the prevention of adverse cardiac events following coronary stenting. Furthermore, a prospective randomized controlled trial revealed that ticlopidine plus aspirin resulted in a significant reduction in subacute thrombosis compared with cilostazol plus aspirin (14).

Therefore, the aim of the present meta-analysis was to compare the differences between cilostazol plus aspirin and ticlopidine plus aspirin with regard to the late restenosis and stent thrombosis rates in patients with CHD following coronary stenting. This may be beneficial in enabling cardiologists to select the anti-platelet therapy method with the greatest efficacy and cost-effectiveness. Furthermore, such knowledge may be further utilized for the accurate determination of treatment strategies for CHD.

\section{Materials and methods}

Literature search strategy. Relevant papers (published from 1998 to March 1, 2013) were identified through a search in Pubmed, Embase, Web of Science and Chinese BioMedicine (CBM) databases using the following terms: ("coronary disease' or 'coronary diseases' or 'disease, coronary' or 'coronary heart disease' or 'heart disease, coronary') and ('stents' or 'stent' or 'drug-eluting stents' or 'bare metal stent' or 'percutaneous coronary intervention') and ('antiplatelet therapy' or 'cilostazol' or 'aspirin' or 'ticlopidine'). This search strategy was performed iteratively until no other relevant articles were found. The references from the eligible articles or textbooks were also reviewed manually to search for other potential studies. Disagreements were resolved through discussions between the authors.

Inclusion and exclusion criteria. The inclusion criteria for the studies included in the present meta-analysis comprised: i) randomized controlled trials focusing on the differences in late restenosis and stent thrombosis between cilostazol plus aspirin and ticlopidine plus aspirin for patients with CHD following coronary stenting; ii) studies with follow-up periods of $>1$ month; iii) studies where the published data concerning the rates of restenosis and stent thrombosis were sufficient; iv) studies published in the English or Chinese languages. Studies were excluded when they were: i) Not clinically-controlled or relevant to the use of cilostazol plus aspirin and ticlopidine plus aspirin for patients with CHD following coronary stenting; ii) duplicates of previous publications; iii) based on incomplete data; iv) case reports, letters, reviews, meta-analyses or editorial articles. If more than one study by the same authors using the same case series was published, either the study with the largest sample size or the most recently published study was selected.

Data extraction. Using a standardized form, data from the studies were extracted independently by two authors. The following information was obtained for each of the studies: First author, year of publication, country, language, study design, numbers of test subjects, eligible lesions, follow-up periods, antiplatelet drug and dose or dosage, and the rates of restenosis and stent thrombosis. In case of conflicting evaluations, an agreement was reached following a discussion between the authors. When required, a third review resolved any discrepancies or uncertainties with regard to the data extraction process.

Quality assessment of the included studies. The methodological quality of each of the included studies was evaluated by two independent reviewers using the Physiotherapy Evidence Database (PEDro) scale (15). Eleven assessment items matching with the quality appraisal were used in this meta-analysis, with scores ranging from 0 to 10 . The PEDro criteria are based on the presence/absence of 11 items: Eligibility criteria, random allocation, allocation concealment, similar baseline characteristics, blinding of all subjects, blinding of therapists, blinding of outcome assessors, crossover rate of $<15 \%$, intention-to-treat analysis, statistical comparisons between groups and measures of variability.

Statistical analysis. The differences in late restenosis and stent thrombosis rates between cilostazol plus aspirin and ticlopidine plus aspirin were measured by odds ratios (ORs) or standardized weight differences (SMDs), with $95 \%$ confidence intervals (CIs). The statistical significance of the pooled value was examined using the $\mathrm{Z}$ test. Interstudy variations and heterogeneities were estimated using the Cochran's Q-statistic, with $\mathrm{P}<0.05$ indicating a statistically significant heterogeneity $(16,17)$. The effect of heterogeneity was also quantified using the $\mathrm{I}^{2}$ test (ranges from 0 to $100 \%$ ), which represented the proportion of interstudy variability that may be contributed to heterogeneity rather than chance. When a significant $\mathrm{Q}$-statistic $(\mathrm{P}<0.05)$ or $\mathrm{I}^{2}>50 \%$ indicated that heterogeneity existed among the studies, the random effects model (DerSimonian Laird method) was conducted for the meta-analysis; otherwise, the fixed effects model (Mantel-Haenszel method) was used. An analysis of sensitivity was performed by omitting each study in turn to assess the quality and consistency of the results. Begger's funnel plots were used to detect publication biases. In addition, the Egger's linear regression test, which measures funnel plot asymmetry using a natural logarithm scale of OR, was used to evaluate the publication biases (18). To ensure the reliability and the accuracy of the results, two authors assessed the data in the statistical software programs independently and obtained the same results. All the P-values were two-sided and all analyses were calculated using Stata statistical software, version 12.0 (Stata Corp., College Station, TX, USA).

\section{Results}

Characteristics of the included studies. According to the inclusion criteria, 12 randomized controlled trials were included in this meta-analysis $(3-5,8,14,19-25)$. The publication year of the included studies ranged from 1999 to 2006. The flow chart of study selection is shown in Fig. 1. The meta-analysis comprised a total of 2,708 patients with CHD following coronary stenting, including 1,371 patients treated with cilostazol plus aspirin and 1,337 patients treated with ticlopidine plus aspirin. The doses of aspirin ranged from 80 to $243 \mathrm{mg} / \mathrm{day}$, 


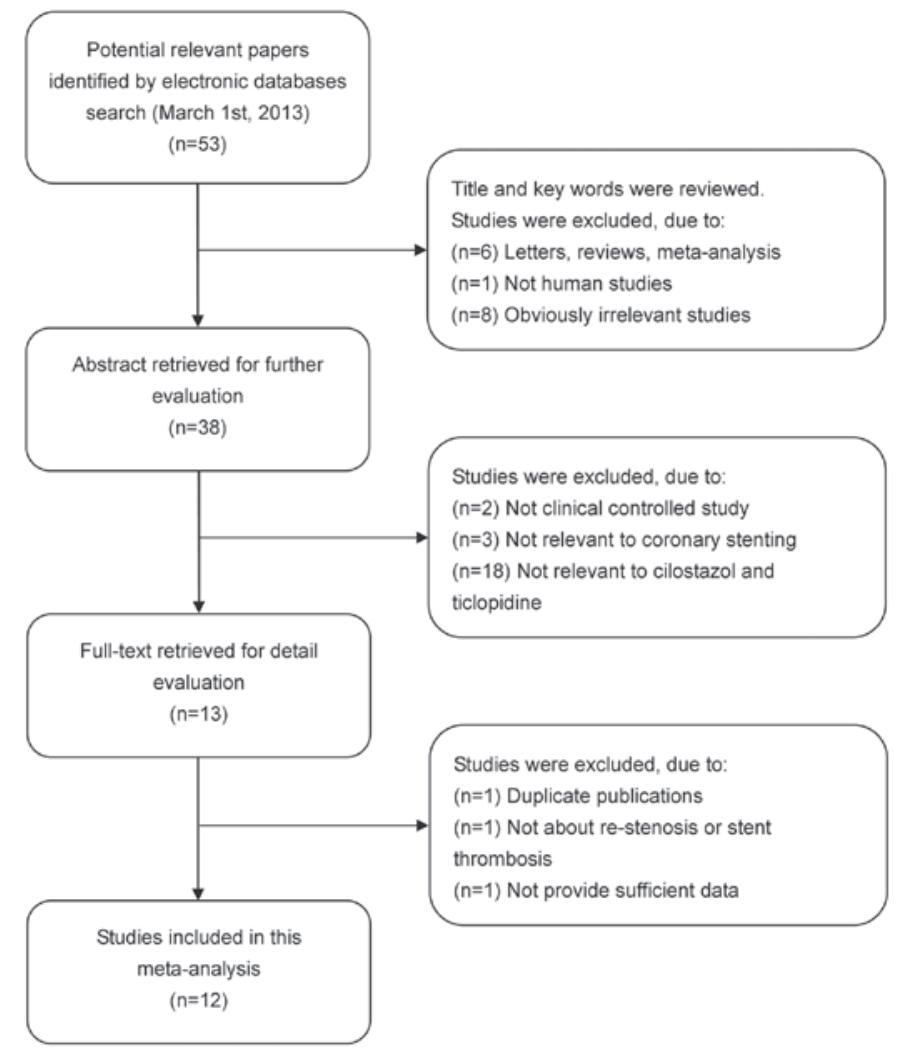

Figure 1. Flow chart of literature search and study selection.

while ticlopidine ranged from 200 to $500 \mathrm{mg} /$ day and cilostazol was administered at $100 \mathrm{mg} / \mathrm{bid}$. The follow-up periods ranged from 1 to 12 months. The main characteristics of all the eligible studies are listed in Table I.

Quantitative data synthesis. Six studies referred to the differences between cilostazol plus aspirin and ticlopidine plus aspirin with regard to the rates of restenosis in patients with CHD following coronary stenting. There was no evident heterogeneity $\left(\mathrm{P}=0.465, \mathrm{I}^{2}=0 \%\right)$, and therefore the fixed effects model was used. When all the eligible studies were pooled into the meta-analysis, the results showed that the patients treated with cilostazol plus aspirin exhibited a lower rate of restenosis than those with ticlopidine plus aspirin $(\mathrm{OR}=0.83$, 95\% CI=0.69-0.99, $\mathrm{P}=0.047$; Fig. 2). Furthermore, a significant difference was observed in the average percent diameter stenosis between cilostazol plus aspirin and ticlopidine plus aspirin $(\mathrm{SMD}=-0.57,95 \% \mathrm{CI}=-0.92--0.23, \mathrm{P}=0.001$; Fig. 3 ).

The difference in the rate of stent thrombosis between cilostazol plus aspirin and ticlopidine plus aspirin was discussed in six studies. Since no significant heterogeneity was observed, the fixed effects model was used. The results of the meta-analysis showed that the incidence of stent thrombosis in patients treated with cilostazol plus aspirin was not significantly lower than that in those treated with ticlopidine plus aspirin $(\mathrm{OR}=1.66,95 \% \mathrm{CI}=0.72-3.80, \mathrm{P}=0.235)$. Furthermore, there were no significant differences in the incidences of acute or subacute stent thrombosis in patients treated with cilostazol plus aspirin compared with those treated with ticlopidine plus aspirin $(\mathrm{OR}=0.98,95 \% \mathrm{CI}=0.14-6.99, \mathrm{P}=0.983 ; \mathrm{OR}=1.85$, 95\% CI=0.73-6.99, $\mathrm{P}=0.467$, respectively; Fig. 4).
Sensitivity analysis and publication bias. A sensitivity analysis was performed to assess the influence of each individual study on the pooled ORs by omitting each of the individual studies in turn. The analysis results suggested that no individual study significantly affected the pooled values of the rates of restenosis or stent thrombosis (Fig. 5), indicating statistically robust results.

Publication bias exists to the extent that the available results for a study are unrepresentative of all the results for that study. Begger's funnel plots and Egger's linear regression tests were performed to assess the publication bias of the included studies. The shapes of the funnel plots did not reveal any indication of obvious asymmetry (Fig. 6). The Egger's tests also showed that there was no statistically significant evidence of publication bias for the rates of restenosis and stent thrombosis $(\mathrm{t}=-2.04, \mathrm{P}=0.111 ; \mathrm{t}=-1.18, \mathrm{P}=0.292$, respectively).

\section{Discussion}

Cilostazol, a selective phosphodiesterase III inhibitor, has been demonstrated to be effective in reducing the incidence of restenosis following coronary stenting (26). At present, the combination of cilostazol and aspirin is regarded as the most acceptable option for the antithrombotic treatment of patients with CHD undergoing coronary stenting (27). However, certain studies have shown that aspirin plus ticlopidine is superior to the combination of cilostazol and aspirin with regard to the midterm occurrence of adverse side-effects (28). Ticlopidine is a potent inhibitor of collagen-induced platelet aggregation, which has been demonstrated to decrease the incidence of clinical events following coronary stenting (14). By activating 


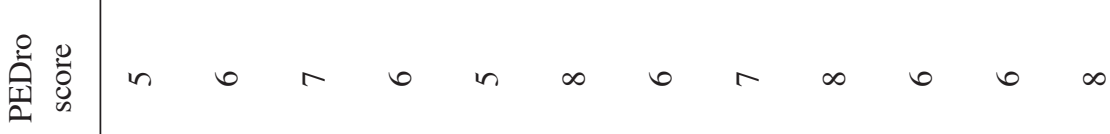

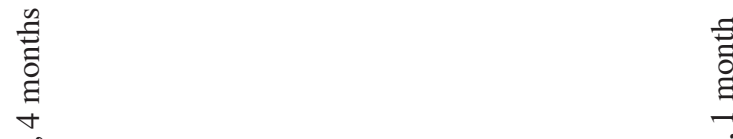

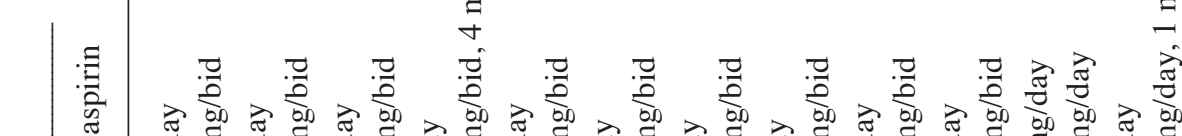

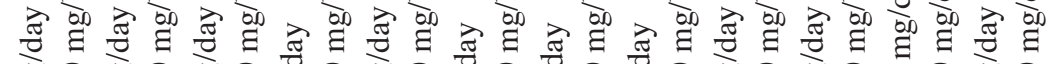

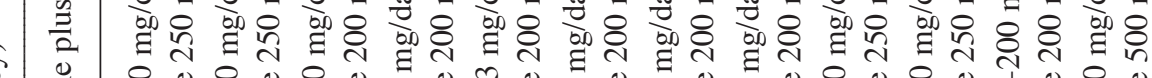

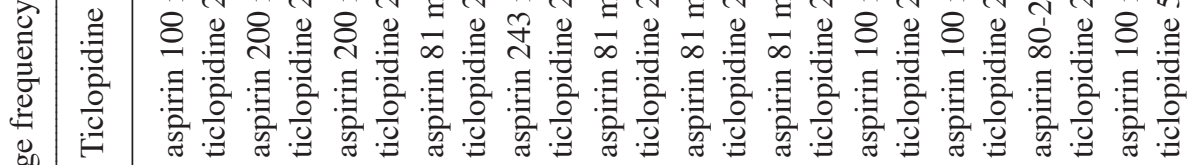

营

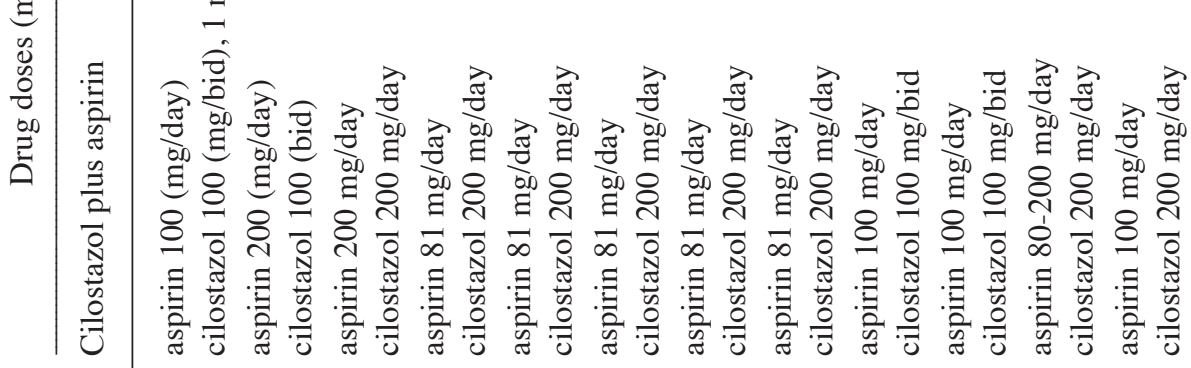

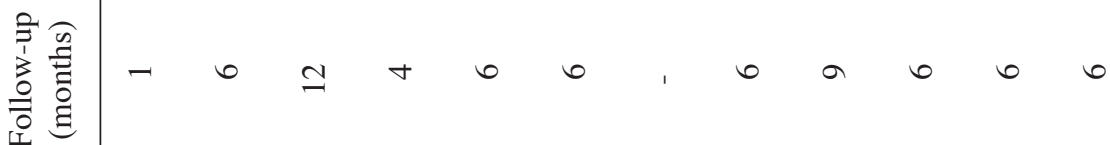

:

吾 है

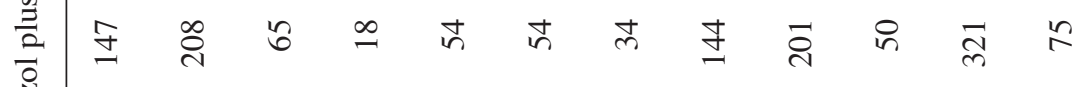

$\stackrel{\frac{\pi}{0}}{\stackrel{0}{0}}$

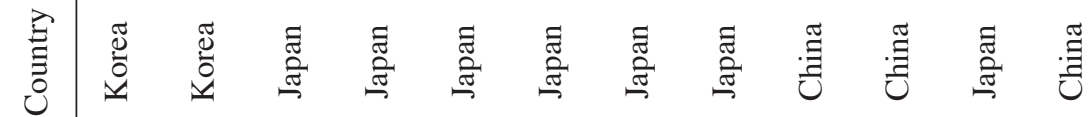

ঠ্خ

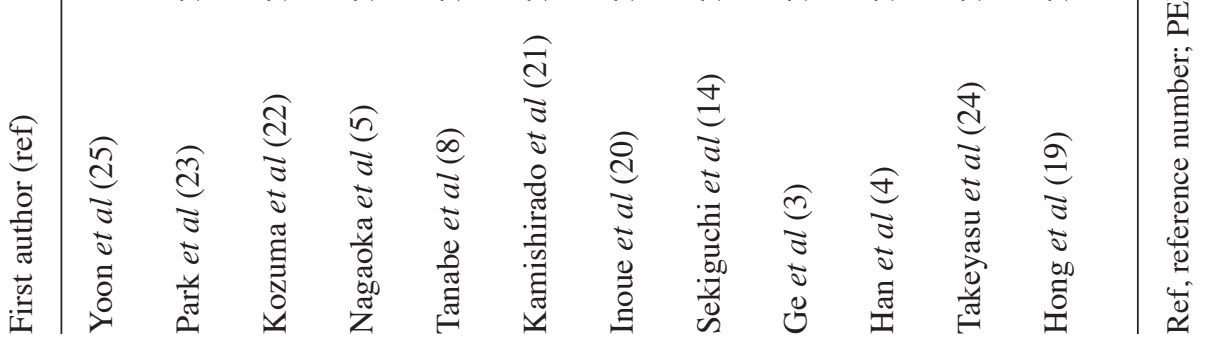




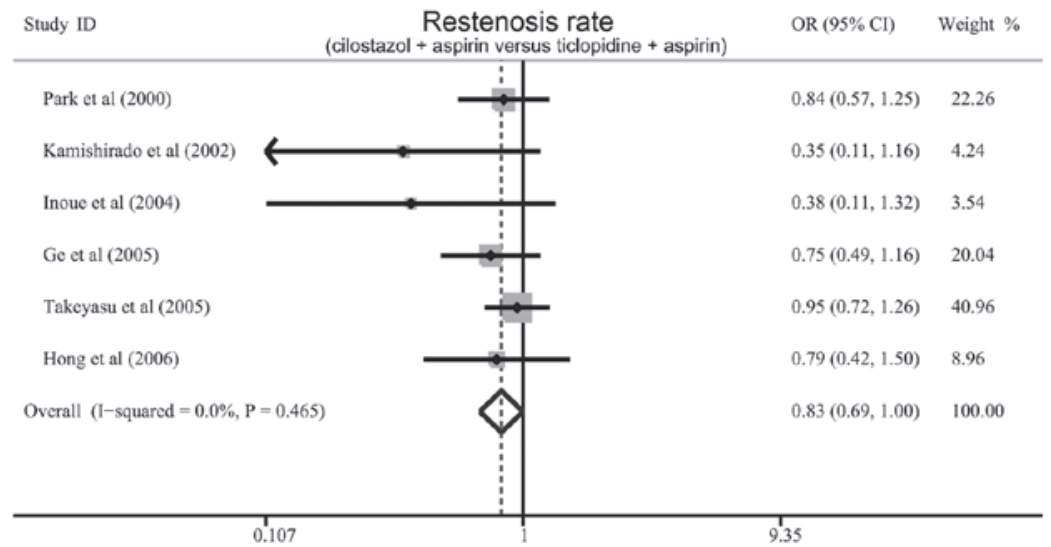

Figure 2. Forest plot of odds ratios (ORs) for the difference in the rate of restenosis between cilostazol plus aspirin and ticlopidine plus aspirin. CI, confidence interval.

\begin{tabular}{|c|c|c|c|}
\hline Study ID & $\begin{array}{l}\text { Percent diameter stenosis } \\
\text { cilostazol + aspirin versus ticlopidine + aspirin) }\end{array}$ & $\operatorname{SMD}(95 \% \mathrm{Cl})$ & Weight $\%$ \\
\hline Park et al (2000) & & $-0.19(-0.36,-0.01)$ & 12.71 \\
\hline Kozuma et al (2001) & & $-0.69(-1.08,-0.30)$ & 11.28 \\
\hline Nagaoka et al (2001) & & $-0.07(-0.64,0.51)$ & 9.70 \\
\hline Tanabe et al (2001) & & $-0.69(-1.06,-0.31)$ & 11.42 \\
\hline Kamishirado et al (2002) & & $-0.51(-0.86,-0.16)$ & 11.61 \\
\hline Inoue et al (2004) & & $-3.89(-4.72,-3.06)$ & 7.53 \\
\hline Sekiguchi et al (2004) & & $-0.10(-0.32,0.12)$ & 12.50 \\
\hline Ge et al (2005) & & $-0.16(-0.35,0.04)$ & 12.61 \\
\hline Han et al (2005) & & $-0.03(-0.50,0.43)$ & 10.64 \\
\hline Overall (1-squared $=91.1 \%, \mathrm{P}<0.001)$ & & $-0.57(-0.92,-0.23)$ & 100.00 \\
\hline NOTE: Wcights are from random effects analysis & & & \\
\hline
\end{tabular}

Figure 3. Forest plot of odds ratios (ORs) for the difference in average percent diameter stenosis between cilostazol plus aspirin and ticlopidine plus aspirin. CI, confidence interval.

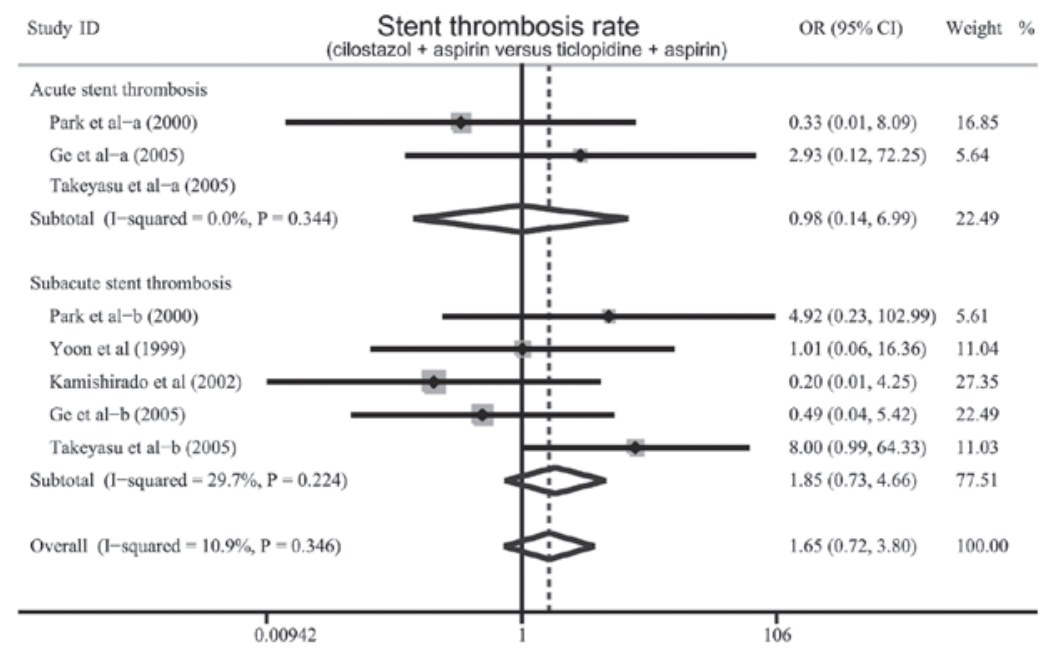

Figure 4. Forest plot of odds ratios (ORs) for the difference in the rates of stent thrombosis between cilostazol plus aspirin and ticlopidine plus aspirin. CI, confidence interval.

platelet adenylate cyclase, ticlopidine is able to enhance the stimulatory action of prostaglandin E1 (PGE1) on the cyclase and block the inhibitory action of PGE2 on the cyclase (5). However, compared with cilostazol, the use of ticlopidine may result in more severe side-effects, therefore leading to a shorter course of treatment (29). Numerous studies have been designed to compare the effectiveness of cilostazol plus aspirin with ticlopidine plus aspirin $(3,5,21)$. However, the definite 
A

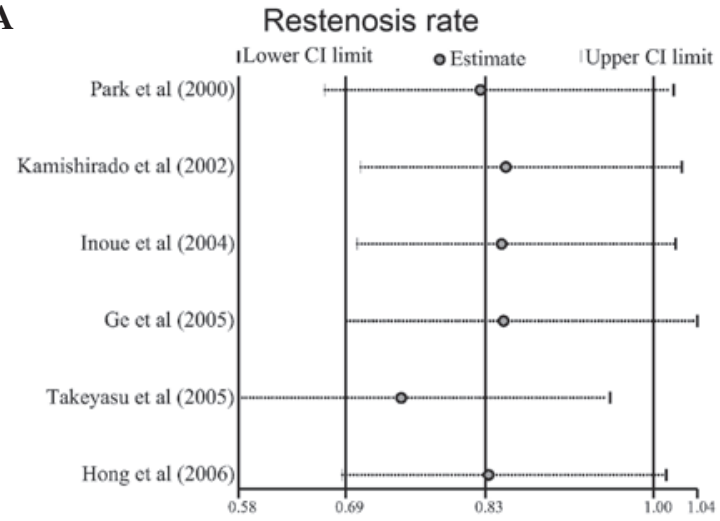

B

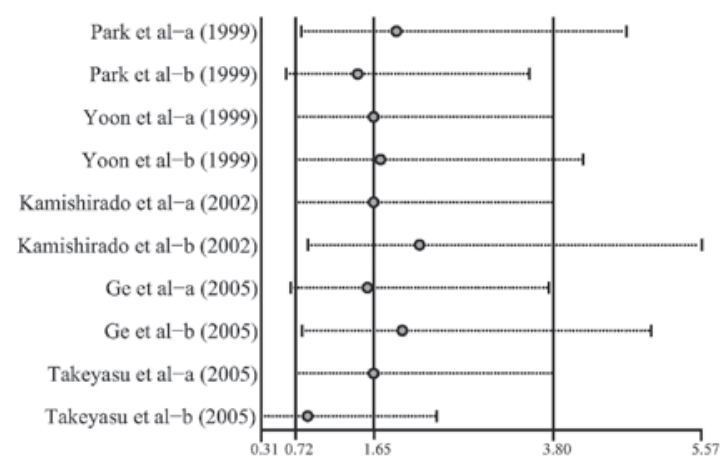

Figure 5. Sensitivity analysis of the summary odds ratio coefficients on (A) the rates of restenosis and (B) stent thrombosis between cilostazol plus aspirin and ticlopidine plus aspirin. ${ }^{a}$ Acute stent thrombosis; ${ }^{b}$ subacute stent thrombosis. Results were computed by omitting each study in turn. Meta-analysis random-effects estimates (exponential form) were used. The two ends of the dotted lines represent the $95 \%$ confidence intervals (CIs).

outcomes of the quantitative angiographic analyses of the two antithrombotic regimens remain in dispute. Thus, there is a requirement for a comprehensive well-defined comparison of the two groups of antithrombotic regimens to be implemented. As a powerful statistical method, a meta-analysis provides a quantitative approach for pooling the results of different studies on the same topic. Therefore, a systematic review and meta-analysis of the differences between cilostazol plus aspirin and ticlopidine plus aspirin, with regard to the rates of restenosis and stent thrombosis, was of great value.

In this meta-analysis, 12 randomized controlled studies were included with a total of 2,708 patients with CHD following coronary stenting. The patient population comprised 1,371 patients treated with cilostazol plus aspirin and 1,337 patients treated with ticlopidine plus aspirin. The predominant finding of this meta-analysis was that the rates of restenosis in patients treated with cilostazol plus aspirin were significantly lower than those in patients treated with ticlopidine plus aspirin, suggesting that cilostazol may be more effective than ticlopidine in reducing restenosis. A possible reason may be the different functional mechanisms of the two antithrombotic agents. However, no significant differences were observed in the rates of acute or subacute stent thrombosis between cilostazol plus aspirin and ticlopidine plus aspirin. These results suggested that there was no difference between cilostazol and ticlopidine with regard to their efficacy as an adjunctive therapy to coronary stenting or in the prevention of stent-associated thrombosis. This is despite the
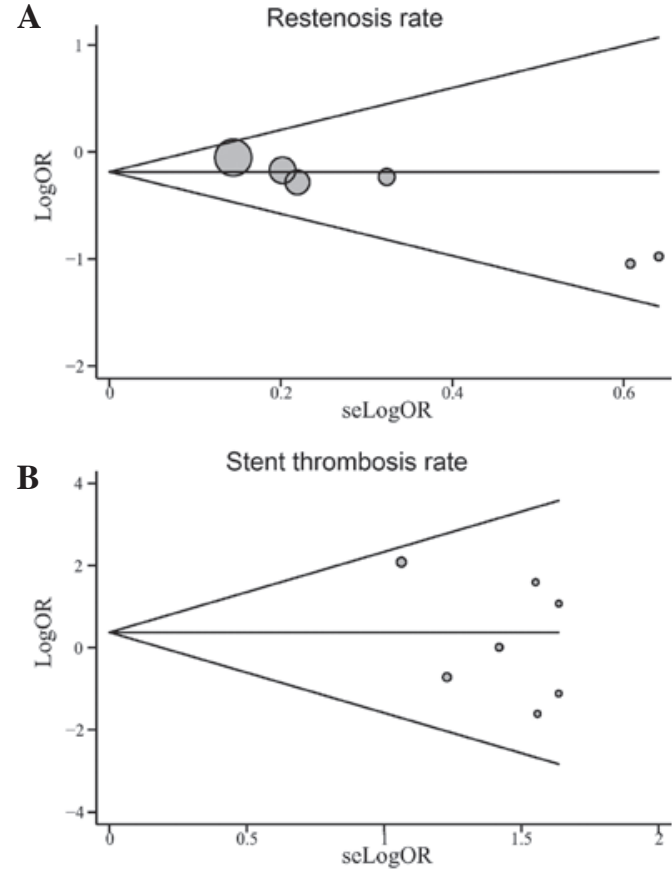

Figure 6. Begger's funnel plot of publication bias in the selected studies on the rates of (A) restenosis and (B) stent thrombosis between cilostazol plus aspirin and ticlopidine plus aspirin. Each point represents a separate study for the indicated correlation. LogOR, natural logarithm of OR; OR, odds ratio; horizontal line, mean magnitude of the effect; seLogOR, standard error of LogOR.

fact that cilostazol demonstrates a different anti-platelet mechanism to ticlopidine, which may lead to a suppression of platelet aggregation. These results were inconsistent with the outcomes published by Hashiguchi et al and Schleinitz et al $(6,29)$, which may be due to the limited number of included studies.

In the interpretation of the results of the present meta-analysis, it is necessary for certain specific issues pertinent to the study to be addressed. The sample size included in the meta-analysis is relatively small and may not provide sufficient statistical power to estimate the differences between cilostazol plus aspirin and ticlopidine plus aspirin. Furthermore, potential heterogeneity and bias may exist due to the differences in the inclusion criteria, follow-up periods, doses of antiplatelet drugs and the severity of disease. In addition, as previously mentioned, each pretreatment regimen was not always identical and the doses of aspirin, ticlopidine or cilostazol were variable. Further limitations included the facts that the type of stent used in each patient was not always identical and there may have been differences with regard to the efficacy of the antiplatelet agents. Moreover, although all participants of each study were well defined with similar inclusion criteria, there may be factors that were not taken into account and that may have influenced our results. There is thus a requirement for the present results to be interpreted with caution due to the potential heterogeneity among trials.

In conclusion, this meta-analysis suggests that the use of cilostazol plus aspirin may result in lower restenosis rates and percent diameter stenosis than ticlopidine plus aspirin for patients with CHD following coronary stenting. However, further well-designed clinical trials are required to investigate the differences between cilostazol and ticlopidine. 


\section{References}

1. Fatima S, Ahmad SI and Ahmad HR: The intercept and slope of breathlessness/chest pain-heart rate relationship in patients with coronary artery disease using exercise tolerance test. J Pak Med Assoc 62: 382-385, 2012.

2. Kang YH, Lao HY, Yu XY, Chen JY and Zhong SL: Progress in genetic and epigenetic research on in-stent restenosis after percutaneous coronary interventions. Zhonghua Yi Xue Yi Chuan Xue Za Zhi 29: 38-42, 2012 (In Chinese).

3. Ge J, Han Y, Jiang H, et al; RACTS (Randomized Prospective Antiplatelet Trial of Cilostazol Versus Ticlopidine in Patients Undergoing Coronary Stenting) Trial Investigators: RACTS a prospective randomized antiplatelet trial of cilostazol versus ticlopidine in patients undergoing coronary stenting: long-term clinical and angiographic outcome. J Cardiovasc Pharmacol 46 : $162-166,2005$

4. Han Y, Wang S, Li Y, et al: Cilostazol improves long-term outcomes after coronary stent implantation. Am Heart J 150 $568,2005$.

5. Nagaoka N, Matsubara T, Okazaki K, Masuda N, Shikaura K and Hotta A: Comparison of ticlopidine and cilostazol for the prevention of restenosis after percutaneous transluminal coronary angioplasty. Jpn Heart J 42: 43-54, 2001.

6. Hashiguchi M, Ohno K, Nakazawa R, Kishino S, Mochizuki M and Shiga T: Comparison of cilostazol and ticlopidine for one-month effectiveness and safety after elective coronary stenting. Cardiovasc Drugs Ther 18: 211-217, 2004.

7. Harding SA, Walters DL, Palacios IF and Oesterle SN: Adjunctive pharmacotherapy for coronary stenting. Curr Opin Cardiol 16: 293-299, 2001

8. Tanabe Y, Ito E, Nakagawa I and Suzuki K: Effect of cilostazol on restenosis after coronary angioplasty and stenting in comparison to conventional coronary artery stenting with ticlopidine. Int J Cardiol 78: 285-291, 2001.

9. Jang JS, Jin HY, Seo JS, et al: A meta-analysis of randomized controlled trials appraising the efficacy and safety of cilostazo after coronary artery stent implantation. Cardiology 122 133-143, 2012.

10. Nakamura T, Tsuruta S and Uchiyama S: Cilostazol combined with aspirin prevents early neurological deterioration in patients with acute ischemic stroke: a pilot study. J Neurol Sci 313: 22-26, 2012.

11. Dangas G, Mehran R, Abizaid AS, et al: Combination therapy with aspirin plus clopidogrel versus aspirin plus ticlopidine for prevention of subacute thrombosis after successful native coronary stenting. Am J Cardiol 87: 470-472, 2001.

12. Rogers KC, Faircloth JM and Finks SW: Use of cilostazol in percutaneous coronary interventions. Ann Pharmacother 46: 839-850, 2012.

13. Geng DF, Liu M, Jin DM, Wu W, Deng J and Wang JF: Cilostazol-based triple antiplatelet therapy compared to dual antiplatelet therapy in patients with coronary stent implantation: a meta-analysis of 5,821 patients. Cardiology 122: 148-157, 2012

14. Sekiguchi M, Hoshizaki H, Adachi H, Ohshima S, Taniguchi K and Kurabayashi M: Effects of antiplatelet agents on subacute thrombosis and restenosis after successful coronary stenting: a randomized comparison of ticlopidine and cilostazol. Circ J 68 610-614, 2004.
15. Maher CG, Sherrington C, Herbert RD, Moseley AM and Elkins M: Reliability of the PEDro scale for rating quality of randomized controlled trials. Phys Ther 83: 713-721, 2003.

16. Higgins JP and Thompson SG: Quantifying heterogeneity in a meta-analysis. Stat Med 21: 1539-1558, 2002

17. Zintzaras E and Ioannidis JP: Heterogeneity testing in meta-analysis of genome searches. Genet Epidemiol 28: 123-137, 2005.

18. Peters JL, Sutton AJ, Jones DR, Abrams KR and Rushton L: Comparison of two methods to detect publication bias in meta-analysis. JAMA 295: 676-680, 2006.

19. Hong B, Qian JY, Fan B, Jin XJ and Ge JB: A randomized trial of cilostazol versus ticlopidine for anti-platelet therapy after coronary artery stenting for prevention of restenosis. Chin J Intervent Cardiol 14: 170-172, 2006 (In Chinese).

20. Inoue T, Uchida T, Sakuma M, et al: Cilostazol inhibits leukocyte integrin Mac-1, leading to a potential reduction in restenosis after coronary stent implantation. J Am Coll Cardiol 44: 1408-1414, 2004.

21. Kamishirado H, Inoue T, Mizoguchi K, et al: Randomized comparison of cilostazol versus ticlopidine hydrochloride for antiplatelet therapy after coronary stent implantation for prevention of late restenosis. Am Heart J 144: 303-308, 2002.

22. Kozuma K, Hara K, Yamasaki M, et al: Effects of cilostazol on late lumen loss and repeat revascularization after Palmaz-Schatz coronary stent implantation. Am Heart J 141: 124-130, 2001.

23. Park SW, Lee CW, Kim HS, et al: Effects of cilostazol on angiographic restenosis after coronary stent placement. Am J Cardiol 86: 499-503, 2000.

24. Takeyasu N, Watanabe S, Noguchi Y, Ishikawa K, Fumikura Y and Yamaguchi I: Randomized comparison of cilostazol vs ticlopidine for antiplatelet therapy after coronary stenting. Circ J 69: 780-785, 2005

25. Yoon Y, Shim WH, Lee DH, et al: Usefulness of cilostazol versus ticlopidine in coronary artery stenting. Am J Cardiol 84: 1375-1380, 1999

26. Jennings DL and Kalus JS: Addition of cilostazol to aspirin and a thienopyridine for prevention of restenosis after coronary artery stenting: a meta-analysis. J Clin Pharmacol 50: 415-421, 2010.

27. Rubboli A, Milandri M, Castelvetri C and Cosmi B: Meta-analysis of trials comparing oral anticoagulation and aspirin versus dual antiplatelet therapy after coronary stenting. Clues for the management of patients with an indication for long-term anticoagulation undergoing coronary stenting. Cardiology 104: 101-106, 2005.

28. Cosmi B, Rubboli A, Castelvetri C and Milandri M: Ticlopidine versus oral anticoagulation for coronary stenting. Cochrane Database Syst Rev: CD002133, 2001.

29. Schleinitz MD, Olkin I and Heidenreich PA: Cilostazol, clopidogrel or ticlopidine to prevent sub-acute stent thrombosis: a meta-analysis of randomized trials. Am Heart J 148: 990-997, 2004. 